\title{
High-energy component of GRB 941017 revisited and the reverse-shock synchrotron self-Compton emission
}

\author{
X. Y. Wang ${ }^{1,2}$, K. S. Cheng ${ }^{1}$, Z. G. Dai ${ }^{2}$, and T. Lu ${ }^{3}$ \\ 1 Department of Physics, The University of Hong Kong, Hong Kong, PR China \\ e-mail: xywang@nju.edu.cn \\ 2 Department of Astronomy, Nanjing University, Nanjing 210093, PR China \\ 3 Purple Mountain Observatory, Chinese Academy of Sciences, Nanjing 210008, PR China
}

Received 4 March 2005 / Accepted 15 April 2005

\begin{abstract}
The different temporal behaviors of the high energy component and the hundreds of keV emission from GRB 941017 suggest that they come from different emission regions. The nearly constant flux of this high energy component is consistent with being produced in the region of the early external shock formed when the ejecta hit the surrounding medium. Here we show that the relatively hard spectrum of this component can be numerically modelled as the synchrotron self-Compton emission from external reverse shock when the ejecta is decelerated by a typical interstellar medium. Constraints on the parameters of the fireball, such as the initial Lorentz factor $\eta$, the burst energy, and the magnetic field equipartition fraction are obtained. Very high initial Lorentz factor $\left(\eta \gtrsim 10^{3}\right)$ inferred for this burst may explain the rarity of this kind of high-energy component in gamma-ray bursts (GRBs). Large GeV-TeV flux from the reverse shock is predicted for GRBs with high initial Lorentz factors.
\end{abstract}

Key words. gamma rays: bursts - radiation mechanisms: non-thermal

\section{Introduction}

In the standard models of gamma-ray bursts, the hundreds of $\mathrm{keV}$ emission arises from internal shocks caused by collisions between the expanding shells due to outflow variability (e.g. Rees \& Mészáros 1994). When the relativistic ejecta interacts with the external medium, a relativistic forward shock expands into the external medium and a reverse shock moves into and heats up the fireball ejecta. The shocked ambient and ejecta materials are in pressure balance and separated by a contact discontinuity. The forward shock continuously heats fresh gas and accelerates electrons, producing long-term afterglows through the synchrotron emission (e.g. Waxman 1997a,b; Wijers et al. 1997). When the reverse shock crosses the shell, the shocked shell and the forward shocked external medium carry a comparable amount of energy. However, the typical temperature of the shocked shell electrons is lower since the particle number density is higher. Thus, the typical frequency of the synchrotron radiation from the shocked shell is considerably lower (Mészáros \& Rees 1993, 1997; Sari \& Piran 1999a). A strong prompt optical flash (Akerlof et al. 1999) and late-time radio flare behavior (Kulkarni et al. 1999), accompanying GRB 990123, have been attributed to the synchrotron emission process from this reverse shock (Sari \& Piran 1999b; Mészáros \& Rees 1999; Nakar \& Piran 2004, 2005; Panaitescu \& Kumar 2004). It was also suggested that reverse shock emission contributes to the early optical afterglows of GRB 021211 (Fox et al. 2003; Wei 2003) and
GRB 021004 (Kobayashi \& Zhang 2003; Zhang et al. 2003; Panaitescu \& Kumar 2004)

In previous papers (Wang et al. 2001a,b), we have studied the inverse Compton (IC) emission from the external shocks, including the synchrotron self-Compton (SSC) processes in GRB forward and reverse shocks, and two combined-IC processes: i.e. scattering of reverse shock photons on the electrons in forward shocks and forward shock photons on the electrons in reverse shocks. We found that the SSC emission from reverse shocks dominates other emission processes in energy bands from tens of $\mathrm{MeV}$ to tens of $\mathrm{GeV}$ for a wide range of shock parameters, and we further suggested that this mechanism may be responsible for the prompt high energy gammarays detected from some bright GRBs by the Energetic Gamma Ray Experiment Telescope (EGRET). Recently, such processes are explored again to see whether it can explain the prompt gamma-ray emission of some GRBs under specific conditions (McMahon et al. 2004) or to study the GeV-TeV emission in the early afterglow (Pe'er \& Waxman 2004b).

González et al. (2003) recently reported the discovery of a distinct high-energy component from GRB 941017, in addition to the usual hundreds of $\mathrm{keV}$ to $\mathrm{MeV}$ components. The lower energy component is well fitted by a band function and has a duration of $T=80 \mathrm{~s}$, over which $90 \%$ of its energy was emitted. The high-energy component, from $\gtrsim 3 \mathrm{MeV}$ up to $\sim 200 \mathrm{MeV}$, displays a roughly constant flux with a relatively hard spectrum $\left(v F_{v} \propto v^{\alpha}\right.$ with $\left.\alpha \sim 1\right)$ and lasts $\lesssim 200 \mathrm{~s}$. 
GRB 941017 is a bright burst with a fluence of about $1.6 \times$ $10^{-4} \mathrm{erg} \mathrm{cm}^{-2}$ (Preece et al. 2000) in the BATSE energy range and has $\gtrsim 3$ times more energy in the high-energy component. The different temporal behaviors suggest that the two components are produced in different regions of the expanding fireball. The similarity of the low-energy component to other bursts suggests that it is produced by internal shocks as usual. The peculiar behavior of the high-energy component is difficult to be accounted for by the synchrotron shock model and has led González et al. (2003) to suggest that it is caused by the hadronic cascades radiation (Waxman 1995; Vietri 1995; Totani 1998; Dermer \& Atoyan 2004). However, as noted by Granot \& Guetta (2003) and by Pe'er \& Waxman (2004a), the high-energy component could be produced by the external shocks when the ejecta begins to be decelerated, as the external shocks can provide the appropriate timescale and the nearly constant temporal behavior.

It was suggested by Granot \& Guetta (2003) that the most probable scenario for this high-energy component is SSC emission from the external reverse shocks. Alternative models are suggested, including IC scattering of the self-absorbed synchrotron photons of the reverse shocks by the electrons in the forward shocks (Pe'er \& Waxman 2004a) and IC scattering of the hundreds of $\mathrm{keV}$ emission by the electrons in the reverse shock at the fireball deceleration radius (Beloborodov 2005). In the former scenario, a wind environment with number density $n \propto r^{-1}$ is favored in order to keep the two break frequencies, $v_{\mathrm{c}}^{\mathrm{IC}}$ and $v_{\mathrm{m}}^{\mathrm{IC}}$, of the inverse Compton component strictly constant in time (i.e. $v_{\mathrm{m}}^{\mathrm{IC}}, v_{\mathrm{c}}^{\mathrm{IC}} \propto t^{0}$ ). They also find that some degree of fine-tuning of the parameters is required. However, we think that a near constancy of the two break frequencies is adequate to explain the observed spectrum, and this could be satisfied for a homogenous-density ISM environment equally well, since the characteristic timescale $(T \sim 80 \mathrm{~s})$ of the reverse shock emission is comparable to the observed durations ( $\$ 200$ s) of the high-energy component.

Motivated by consideration that a homogenous-density ISM environment is more plausible, we investigated whether the SSC emission from the reverse shock for a GRB exploding in a typical ISM could explain the high-energy component of GRB 941017. In Sect. 2, we first analytically derive the constraints on the parameters if the high-energy component is explained as SSC emission from the reverse shock. Because the IC spectral shape is no longer well-described by simple powerlaw approximations at the high-energy frequencies (Sari \& Esin 2001), we also perform a numerical calculation of the IC spectrum and attempt to fit the spectral data of this component in Sect. 3. Finally, our conclusions are summarized in Sect. 4.

\section{Analytic treatment and constraints on the model parameters}

For a long burst of $T \sim 80 \mathrm{~s}$, the reverse shock generally belongs in the "thick shell" category with $\Delta>l / 2 \eta^{8 / 3}$ (Sari \& Piran 1995), where $\Delta=c T$ is the shell width, $l=$ $\left(3 E / 4 \pi n_{1} m_{\mathrm{p}} c^{2}\right)^{1 / 3}$ the Sedov length, and $\eta$ the initial Lorentz factor of the ejecta. In this case, the reverse shock is relativistic, and it considerably decelerates the shell material
(Kobayashi 2000) before it crosses the shell. The characteristic timescale of the reverse shock emission is then the crossing time $\sim \Delta / c=T$.

The Lorentz factor of the shocked shell, after the reverse shock crosses it, is

$\gamma_{\mathrm{A}}=\left(\frac{17 E}{128 \pi n m_{\mathrm{p}} c^{5} T^{3}}\right)^{1 / 8}=180 E_{54}^{1 / 8} n_{0}^{-1 / 8} T_{2}^{-3 / 8}$,

and the Lorentz factor of the reverse shock is thus

$\bar{\gamma}=\eta / 2 \gamma_{\mathrm{A}}=3 \eta_{3} E_{54}^{-1 / 8} T_{2}^{3 / 8} n_{0}^{1 / 8}$,

where $E$ is the isotropic kinetic energy of the burst, $n$ the number density of the ISM, and $m_{\mathrm{p}}$ the proton mass. Unless specified otherwise, $Q_{x}=Q / 10^{x}$ in cgs units.

The distributions of newly-shocked electrons in the reverse shocks are assumed to be a power law of index $p$ $\left(N(\gamma) \propto \gamma^{-p}\right)$ with the minimum Lorentz factor of the random motion of electrons in the shell rest frame being $\gamma_{\mathrm{m}}=$ $(p-2) /(p-1)\left(m_{\mathrm{p}} / m_{\mathrm{e}}\right) \epsilon_{\mathrm{e}} \bar{\gamma}$, where $\epsilon_{\mathrm{e}}$ is the fraction of thermal energy carried by electrons. Assuming that $\epsilon_{\mathrm{B}}$ is the fraction of the thermal energy carried by the magnetic field, the magnetic field strength is $B^{\prime}=12 \mathrm{G} \epsilon_{\mathrm{B},-2}{ }^{1 / 2}\left(\gamma_{\mathrm{A}} / 300\right) n_{0}^{1 / 2}$. The shocked relativistic electrons cool through both synchrotron emission and IC scatterings of the synchrotron photons. The Compton parameter $Y$, defined as the ratio between the IC luminosity of the electrons and the synchrotron luminosity, is $\simeq\left(\epsilon_{\mathrm{e}} / \epsilon_{\mathrm{B}}\right)^{1 / 2}$ if the electrons are in the fast-cooling regime and $\epsilon_{\mathrm{e}} \gg \epsilon_{\mathrm{B}}$, which is well satisfied for the thick shell case we discuss here.

Beloborodov (2005) argues that electrons accelerated in the reverse shock quickly lose their energy by Compton upscattering of the prompt gamma-rays as the observed optical flash of GRB 990123 overlaps with the prompt gamma-ray emission. However, for the high-energy tail of GRB 941017, we think that the cooling in this process is insignificant in comparison with cooling by the SSC emission, because at the tail time, the prompt $\mathrm{MeV}$ emission has decayed to a very low level (González et al. 2003).

Thus we can obtain the two characteristic Lorentz factors, $\gamma_{\mathrm{m}}$ and $\gamma_{\mathrm{c}}$ (the Lorentz factor of the electrons that cool on a timescale equal to the dynamic timescale)

$$
\begin{aligned}
\gamma_{\mathrm{m}} & =1700 f_{\mathrm{p}} \epsilon_{\mathrm{e}, 0.5} \eta_{3} E_{54}^{-1 / 8} T_{2}^{3 / 8} n_{0}^{1 / 8} \\
\gamma_{\mathrm{c}} & =\frac{2000}{Y+1} \epsilon_{\mathrm{B},-2}^{-1} E_{54}^{-\frac{3}{8}} n_{0}^{-\frac{5}{8}} T_{2}^{\frac{1}{8}} \\
& \simeq 250 \epsilon_{\mathrm{e}, 0.5}^{-\frac{1}{2}} \epsilon_{\mathrm{B},-2}^{-\frac{1}{2}} E_{54}^{-\frac{3}{8}} n_{0}^{-\frac{5}{8}} T_{2}^{\frac{1}{8}}
\end{aligned}
$$

where $f_{\mathrm{p}} \equiv 3(p-2) /(p-1)$ and $\epsilon_{\mathrm{e}, 0.5}=\epsilon_{\mathrm{e}} / 0.5$.

As described by Sari et al. (1998), synchrotron radiation from the shocked electrons can be approximated by a broken power-law spectrum with three characteristic break frequencies. One is the self-absorption frequency, $v_{\mathrm{a}}$. The other two are the peak frequencies of the emission from the electrons with the characteristic Lorentz factor $\gamma_{\mathrm{m}}$ and $\gamma_{\mathrm{c}}$, denoted as $v_{\mathrm{m}}$ and $v_{\mathrm{c}}$, respectively. The break frequencies $v_{\mathrm{m}}$ and $v_{\mathrm{c}}$ and peak flux of the synchrotron emission from the reverse shock are respectively given by

$v_{\mathrm{m}}=5.6 \times 10^{15} f_{\mathrm{p}}^{2} \epsilon_{\mathrm{e}, 0.5}^{2} \epsilon_{\mathrm{B},-2}^{1 / 2} \eta^{3} n_{0}^{1 / 2} \mathrm{~Hz}$, 
$v_{\mathrm{c}}=2.4 \times 10^{15}(Y+1)^{-2} \epsilon_{\mathrm{B},-2}^{-3 / 2} E_{54}^{-1 / 2} n_{0}^{-1} T_{2}^{-1 / 2} \mathrm{~Hz}$,

$(Y+1) v_{\mathrm{m}} F_{v_{\mathrm{m}}}=8.7 \times 10^{-7} f_{\mathrm{p}} \epsilon_{\mathrm{e}, 0.5} E_{54} T_{2}^{-1} d_{\mathrm{L}, 28} \mathrm{erg} \mathrm{cm}^{-2} \mathrm{~s}^{-1}$.

The synchrotron self-absorption frequency $v_{\mathrm{a}}$ can be obtained by using Eqs. (9) and (10) in Wang et al. (2001b) and is found to be much lower than $v_{\mathrm{c}}$.

Now we can get the power-law approximation spectrum of the SSC component, whose break frequencies and peak flux are given by

$\nu_{\mathrm{m}}^{\mathrm{IC}}=2 \gamma_{\mathrm{m}}^{2} \nu_{\mathrm{m}}=3.1 \times 10^{22} f_{\mathrm{p}}^{4} \epsilon_{\mathrm{e}, \frac{1}{2}}^{4} \epsilon_{\mathrm{B},-2}^{\frac{1}{2}} \eta_{3}^{4} E_{54}^{-\frac{1}{4}} n_{0}^{\frac{3}{4}} T_{2}^{\frac{3}{4}} \mathrm{~Hz}$,

$\nu_{\mathrm{c}}^{\mathrm{IC}}=2 \gamma_{\mathrm{c}}^{2} \nu_{\mathrm{c}}=6.3 \times 10^{18} \epsilon_{\mathrm{e}, \frac{1}{2}}^{-2} \epsilon_{\mathrm{B},-2}^{-\frac{3}{2}} E_{54}^{-\frac{5}{4}} n_{0}^{-\frac{9}{4}} T_{2}^{-\frac{1}{4}} \mathrm{~Hz}$,

$$
\begin{aligned}
v_{\mathrm{m}}^{\mathrm{IC}} F_{v_{\mathrm{m}}} & =Y v_{\mathrm{m}} F_{v_{\mathrm{m}}} \\
& =8.7 \times 10^{-7} f_{\mathrm{p}} \epsilon_{\mathrm{e}, 0.5} E_{54} T_{2}^{-1} d_{\mathrm{L}, 28} \mathrm{erg} \mathrm{cm}^{-2} \mathrm{~s}^{-1} .
\end{aligned}
$$

The high-energy component of GRB 941017 carries at least $\sim 3$ times more energy than the hundreds of $\mathrm{keV}$ emission. This suggests that the SSC spectrum is very likely to be in the fastcooling regime in order to have a high radiative efficiency, so the SSC spectrum is given by

$v F_{v}^{\mathrm{IC}}=v_{\mathrm{m}}^{\mathrm{IC}} F_{v_{\mathrm{m}}}\left\{\begin{array}{l}\left(v_{\mathrm{c}}^{\mathrm{IC}} / v_{\mathrm{m}}^{\mathrm{IC}}\right)^{1 / 2}\left(v / v_{\mathrm{c}}^{\mathrm{IC}}\right)^{4 / 3} \quad v<v_{\mathrm{c}}^{\mathrm{IC}} \\ \left(v / v_{\mathrm{m}}^{\mathrm{IC}}\right)^{1 / 2} v_{\mathrm{c}}^{\mathrm{IC}}<v<v_{\mathrm{m}}^{\mathrm{IC}} \\ \left(v / v_{\mathrm{m}}^{\mathrm{IC}}\right)^{(2-p) / 2} \quad v_{\mathrm{m}}^{\mathrm{IC}}<v\end{array}\right.$.

The spectrum of the high-energy tail of GRB 941017 has the form of $v F_{v} \sim v$ from a few Mev up to $\sim 200 \mathrm{MeV}$. As suggested by Granot \& Guetta (2003), a possible approach to bring the spectral slope close to $\alpha \sim 1$ is to require $h v_{\mathrm{c}}^{\mathrm{IC}}$ close to tens of $\mathrm{MeV}$, so that $\alpha$ would gradually change from $4 / 3$ to $1 / 2$ in the nearby energy band. The peak energy of the high-energy component is at least $\sim 150 \mathrm{MeV}$, and the corresponding flux is $\gtrsim 2 \times 10^{-6} \mathrm{erg} \mathrm{cm}^{-2} \mathrm{~s}^{-1}$, so we have the following constraints:

$10 \mathrm{MeV} \lesssim h v_{\mathrm{c}}^{\mathrm{IC}} \lesssim 100 \mathrm{MeV}$

$v_{\mathrm{m}}^{\mathrm{IC}} \gtrsim 150 \mathrm{MeV}$

$v_{\mathrm{m}}^{\mathrm{IC}} F_{v_{\mathrm{m}}^{\mathrm{IC}}} \gtrsim 2 \times 10^{-6} \mathrm{erg} \mathrm{cm}^{-2} \mathrm{~s}^{-1}$.

By simple algebraic computation, we finally get

$4 \times 10^{-5} \epsilon_{\mathrm{e}, 0.5}^{-4 / 3} E_{54}^{-5 / 12} n_{0}^{-3 / 2} \lesssim \epsilon_{\mathrm{B}} \lesssim 2 \times 10^{-4} \epsilon_{\mathrm{e}, 0.5}^{-4 / 3} E_{54}^{-5 / 12} n_{0}^{-3 / 2}$

$\eta \gtrsim 1700 f_{\mathrm{p}}^{-1} \epsilon_{\mathrm{e}, 0.5}^{-5 / 6} E_{54}^{1 / 6}$

$E_{54} \gtrsim 2 f_{\mathrm{p}}^{-1} \epsilon_{\mathrm{e}, 0.5}^{-1} d_{\mathrm{L}, 28}^{2}$

for $T_{2}=0.8$. It is remarkable to find that the initial Lorentz factor is independent of the number density of the surrounding medium and very weakly dependent on the burst energy $E$. Since $\epsilon_{\mathrm{e}, 0.5} \lesssim 1$, we get

$\eta \gtrsim 1700$

for a typical value of $p=2.5$. In the same way, the burst energy is constrained to be $E_{54} d_{\mathrm{L}, 28}^{-2} \gtrsim 2$. The constraint on the magnetic field equipartition factor depends on the number density of the surrounding medium. For $E=10^{54} \mathrm{erg}, \epsilon_{\mathrm{e}}=0.5$, and a typical ISM with $n \sim 0.1 \mathrm{~cm}^{-3}$, we get $10^{-3} \lesssim \epsilon_{\mathrm{B}} \lesssim 0.003$.

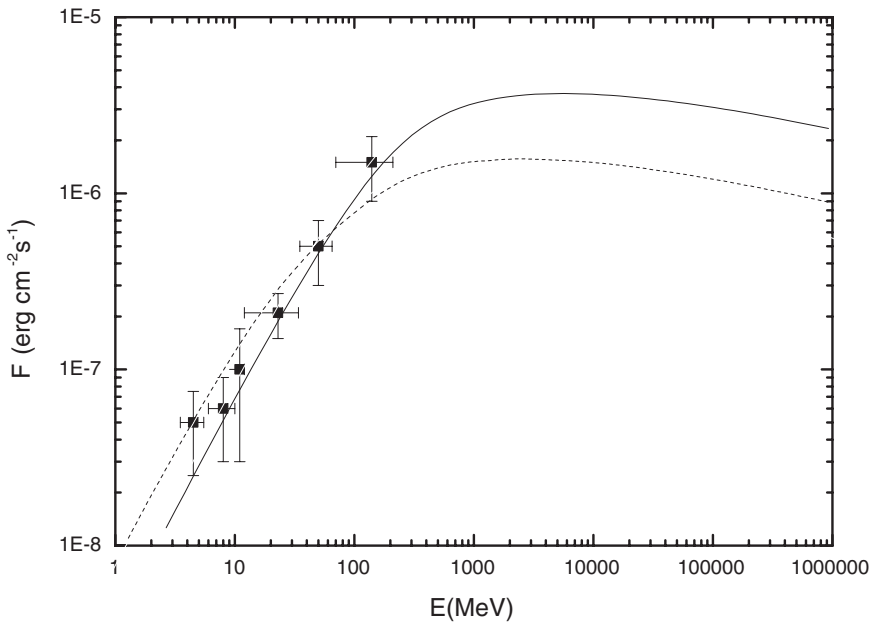

Fig. 1. Fits of the high-energy spectral data of GRB 941017 with the model of the synchrotron self-Compton emission from the reverse shock. Points with error bars are the observed spectrum of GRB 941017 from $\sim 3 \mathrm{MeV}$ up to $200 \mathrm{MeV}$ averaged over a time interval of 100-200 following the burst trigger (González et al. 2003). The two curves have the same model parameters of $E=10^{54} \mathrm{erg}$, $\gamma_{0}=2500, \epsilon_{\mathrm{e}}=0.5, p=2.5$, and $n=0.1 \mathrm{~cm}^{-3}$, but with $\left\{d_{\mathrm{L}}=2.1 \times\right.$ $\left.10^{27} \mathrm{~cm}(z=0.15), \epsilon_{\mathrm{B}}=10^{-3}\right\}$ for the solid line and $\left\{d_{\mathrm{L}}=3.6 \times\right.$ $\left.10^{27} \mathrm{~cm}(z=0.25), \epsilon_{\mathrm{B}}=0.003\right\}$ for the dashed line respectively.

\section{Numerical computation of the SSC spectrum and fit of GRB 941017}

The above analytic treatment makes a basic assumption that the SSC spectrum follows the simple power-law approximation described by Eq. (11). However, we should note that at the highenergy band $\left(v>v_{\mathrm{c}}^{\mathrm{IC}}\right)$, the additional logarithmic terms become important and the simple power-law approximation may not be adequate (Sari \& Esin 2001; Wang et al. 2001b). Thus, we will follow the numerical treatment of the SSC spectrum in Wang et al. (2001b) and apply it to the fit of the high-energy spectrum of GRB 941017.

As derived in Sari \& Esin (2001) and Wang et al. (2001b), the inverse Compton emission flux in the Thomson scattering regime is given by

$F_{v}^{\mathrm{IC}}=3 \Delta r^{\prime} \sigma_{\mathrm{T}} \int_{\gamma_{\min }}^{\gamma_{\max }} \mathrm{d} \gamma N(\gamma) \int_{0}^{1} \mathrm{~d} x g(x) F_{\nu}(x)$

where $\Delta r^{\prime}=\Delta \gamma_{\mathrm{A}} /(4 \bar{\gamma}+3)$ is the comoving width of the shocked shell; $F_{v}$ is the synchrotron emission flux; $g(x)=$ $1+x+2 x \ln (x)-2 x^{2}$ reflects the angular dependence of the scattering cross section for $\gamma_{\mathrm{e}} \gg 1$ (Blumenthal \& Gould 1970); $\sigma_{\mathrm{T}}$ is the Thomson scattering cross section; $\gamma_{\min }$ and $\gamma_{\max }$ are, respectively, the minimum and maximum Lorentz factors of the shock-accelerated electrons in the reverse shock region. The distribution of the fast-cooling electrons is given by

$N(\gamma)=K\left\{\begin{array}{ll}\gamma^{-2} & \text { if } \gamma_{\mathrm{c}}<\gamma<\gamma_{\mathrm{m}} \\ \gamma^{-p-1} \gamma_{\mathrm{m}}^{p-1} & \text { if } \gamma_{\mathrm{m}}<\gamma<\gamma_{\max }\end{array}\right.$,

with $K=n_{\mathrm{r}} \gamma_{\mathrm{c}}$, where $n_{\mathrm{r}}=(4 \bar{\gamma}+3) E /\left(4 \pi m_{\mathrm{p}} c^{2} \eta \gamma_{\mathrm{A}} \Delta r^{2}\right)$ is the number density of the reversely shocked shell and $r=\gamma_{\mathrm{A}}^{2} c T$ the shell radius when the reverse shock crosses the shell. 
In Fig. 1, we present the fit of the high-energy spectral data of GRB 941017 with Eq. (15). We find that the combination of the following parameters can give a reasonably good fit (solid line): $E=10^{54} \mathrm{erg}, \eta=2500, p=2.5, n=0.1 \mathrm{~cm}^{-3}, \epsilon_{\mathrm{e}}=$ $0.5, \epsilon_{\mathrm{B}}=10^{-3}$, and $d_{\mathrm{L}}=2.08 \times 10^{27} \mathrm{~cm}(z=0.15)$. The dashed line has a higher value for $\epsilon_{\mathrm{B}}\left(\epsilon_{\mathrm{B}}=0.003\right)$, hence a lower $v_{\mathrm{c}}^{\mathrm{IC}}$, which causes a slightly flatter spectrum. From the computed spectrum, we can see that a large flux still exists at higher energy spectral band after the peak, which implies that the numerical treatment puts a more stringent constraint on the burst energy $E$ than the analytic one above (Eq. (13)). The solid line and dashed line have $E_{54} d_{\mathrm{L}, 28}^{-2}=23$ and $E_{54} d_{\mathrm{L}, 28}^{-2}=7.7$, respectively.

Such a bright high-energy component appears to be quite rare in GRBs and was seen in only one out of 26 BATSE bursts that were bright at hundreds of $\mathrm{keV}$ and were also detected with EGRET's Total Absorption Shower Counter. The other 25 GRBs showed a high-energy emission that is consistent with the single spectral component observed by BATSE. Owing to the above constraints of $\eta \gtrsim 1700$ obtained for GRB 941017, we speculate that the very high initial Lorentz factor may cause the rarity of the high-energy component in bright GRBs. Very high $\eta$ is expected to have two effects on the high-energy emission: 1) it causes a relativistic reverse shock and the accelerated electrons are in the fast-cooling regime, and therefore have a high radiative efficiency for the SSC emission. For lower initial Lorentz factor (e.g. $\eta<500$ ), the electrons are slow-cooling instead, so the SSC emission flux should become correspondingly lower; 2) the peak of the SSC emission is sensitive to $\eta$ $\left(v_{\mathrm{m}}^{\mathrm{IC}} \propto \eta^{4}\right)$. When $\eta$ becomes smaller, the peak shifts to the lower energy. We plot the SSC emission spectra for different $\eta$ in Fig. 2. The figure shows that when other parameters are the same, the peak energy for $\eta=100$ is at $\sim 10 \mathrm{MeV}$ and the peak flux is one order of magnitude lower than the case for $\eta=2500$. This may explain why high-energy components such as GRB 941017 are not observed for most bright GRBs.

From Fig. 2 we also can see that for higher $\eta$, the $\mathrm{GeV}-\mathrm{TeV}$ flux is larger. The sensitivity of future high-energy detector Gamma-Ray Large Area Space Telescope (GLAST) at the energy $10-300 \mathrm{GeV}$ is also plotted for comparison, assuming that at least five photons should be detected and an averaged effective area of $\sim 10000 \mathrm{~cm}^{2}$ for GLAST Large Area Telescope at this energy range ${ }^{1}$. It has been shown that due to the relatively large size of the deceleration radius where the reverse shocks occur, the high-energy cutoff due to the internal absorption of high energy gamma-rays by pair-production is greater than $\gtrsim 1 \mathrm{TeV}$ (Wang et al. 2004). The attenuation due to interaction with cosmic infrared photons may be significant for high-energy photons with energies larger than $\sim 500 \mathrm{GeV}$ for GRBs at $z \gtrsim 0.3$, but will not affect $\mathrm{GeV}$ to sub$\mathrm{TeV}$ photons (e.g. de Jager \& Stecker 2002; Aharonian et al. 2002). Thus we expect the $\mathrm{GeV}$ to sub-TeV emission could be well detected by future high-energy detector such as GLAST and ground-based sub-TeV detectors, especially when $\eta$ is as high as $\sim 1000$. Another prediction of the SSC model is that there would be temporal correlation between the high-energy

\footnotetext{
${ }^{1}$ See http://www-glast.slac.stanford.edu
}

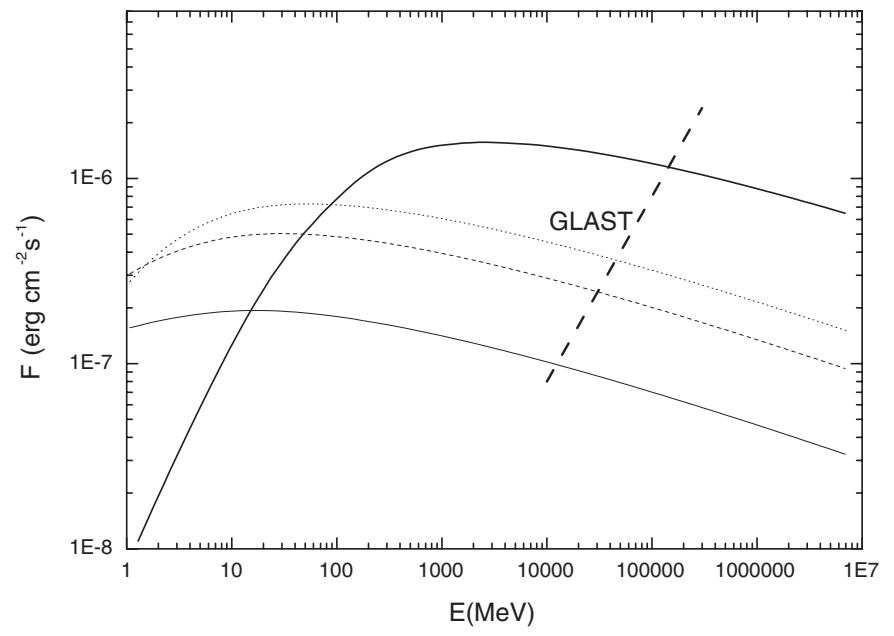

Fig. 2. Comparison of the energy spectra of the SSC emission from the reverse shock for different values of the initial Lorentz factors of the fireball ejecta. The four lines have the same model parameters of $E=10^{54} \mathrm{erg}, n=0.1 \mathrm{~cm}^{-3}, \epsilon_{\mathrm{e}}=0.5, \epsilon_{\mathrm{B}}=0.003, p=2.5$, and $d_{\mathrm{L}}=3.6 \times 10^{27} \mathrm{~cm}(z=0.25)$, but with different values of $\eta: \eta=$ 100 (thin solid line), $\eta=300$ (dashed line), $\eta=500$ (dotted solid line), and $\eta=2500$ (thick solid line). The solid dashed line shows the sensitivity of future high-energy detector Gamma-Ray Large Area Space Telescope (GLAST) at the energy 10-300 GeV, assuming at least five photons should be detected.

component and the synchrotron component (Wang et al. 2003) that usually displays as a bright optical-UV flash as observed from GRB 990123. Both these predictions could be tested in the upcoming Swift-GLAST era.

\section{Discussion and conclusions}

Owing to the highly variable time structure, the hundreds of $\mathrm{keV}$ component of GRBs is widely believed to come from the region of the internal shock caused by the collisions between the shells due to the outflow variability. But the detailed emission process for this prompt emission is still being debated, and one of the unsettled problems is the clustering of the peak energy of this emission (see Zhang \& Mészáros 2002, for detailed discussions). The proposed models include the synchrotron emission or the SSC emission mechanisms of the shock-accelerated electrons (Panaitescu \& Mészáros 2000), the emission from the optically thick photosphere due to the presence of abundant secondary electrons (Mészáros et al. 2002), etc. If the hundreds of $\mathrm{keV}$ emission is produced by the synchrotron emission, the peak energy should be given by

$E_{\text {peak }}=h v_{\mathrm{m}}^{\text {syn }}=10 f_{\mathrm{p}}^{2} \epsilon_{\mathrm{e}, 0.5}^{2} \epsilon_{\mathrm{B},-2}^{1 / 2}\left(\gamma_{\mathrm{in}} / 2\right)^{2} \eta_{3}^{-2} E_{54} t_{\mathrm{v},-3}^{-1} \mathrm{keV}$

where $\gamma_{\text {in }}$ is the Lorentz factor of the internal shock and $t_{\mathrm{v}}$ the variability time of the outflow (e.g. Dai \& Lu 2002; Guetta \& Granot 2003). So, for $\eta=2000, \epsilon_{\mathrm{e}}=0.5$, and $\gamma_{\text {in }}=2$, we need a short variability time $t_{\mathrm{v}}=10^{-4} \mathrm{~s}$ and $E_{54} \epsilon_{\mathrm{B},-2}>16$ to have $h v_{\mathrm{m}}^{\text {syn }} \sim 100 \mathrm{keV}$ for GRB 941017. According to Eq. (13), if we take $\epsilon_{\mathrm{B}} \sim 0.1$, we will need a low density medium with $n \sim 0.01 \mathrm{~cm}^{-3}$.

Our work differs from earlier ones in the following aspects: 1) a wind environment with number density $n \propto r^{-1}$ 
was suggested by Granot \& Guetta (2003) in order to keep the two break frequencies, $v_{\mathrm{c}}^{\mathrm{IC}}$ and $\nu_{\mathrm{m}}^{\mathrm{IC}}$, strictly constant in time. Such a wind environment seems unnatural to GRBs and they also find that some degree of fine-tuning of the parameters is required to explain the high-energy spectrum in this case. This led them to suggest that some additional or alternative emission mechanism might be at work. However, we argue that the near constancy of the two break frequencies, which could be satisfied by a homogenous-density ISM environment quite well, is adequate enough to explain the observed spectrum. Such a homogenous-density ISM environment is more probable than the $n \propto r^{-1}$ wind environment for GRBs. Moreover, reasonable model parameters for explaining the high-energy spectrum are found for the homogenous-density ISM environment. As a result, we think that the high-energy component of GRB 941017 could be entirely explained by the SSC emission from the reverse shocks, the same process studied in detail by Wang et al. (2001b) and invoked to explain the prompt high energy gamma-rays detected from some bright GRBs by the Energetic Gamma Ray Experiment Telescope (EGRET) (Wang et al. 2001a); 2) we performed a numerical calculation of the high-energy spectrum of the reverse-shock SSC emission, which is very necessary as the IC spectral shape could no longer be described by simple power-law approximations as used in the analytic treatment at the high-energy frequencies $v>v_{\mathrm{c}}^{\mathrm{IC}}$ (Sari \& Esin 2001). It was found that the numerical calculation gives more stringent constraints on the model parameters than the simple analytic treatment. We also obtained a reasonably good fit of the high-energy spectral date of GRB 941017;3) we numerically investigate the effects of the value of the initial Lorentz factor on the high-energy spectrum of the SSC emission from the reverse shocks. Figure 2 shows clearly that the rarity of the high-energy component as GRB 941017 can be caused by a high value of the initial Lorentz factor.

In summary, we have shown that the flat spectrum of the high-energy tail of GRB 941017 can be reproduced by the SSC emission from the reverse shock formed when the ejecta interacts with a homogenous interstellar medium. The reverse shock is favored to be fast-cooling so as to have high radiative efficiency for this SSC emission. In this scenario, the relatively high peak energy and hard spectrum of the high-energy component suggests a large initial Lorentz factor for the GRB ejecta, which in turn may explain the rarity of such a high-energy component in GRBs.

Acknowledgements. We would like to thank A. Pe'er and M. M. González for help on the observation data of GRB 941017. This work was supported by the Special Funds for Major State Basic Research Projects, the National 973 Project, the National Natural Science Foundation of China under grants 10403002, 10233010, and 10221001, the Foundation for the Author of National Excellent
Doctoral Dissertation of PR China and by an RGC grant (project HKU 7014/04P) of the Hong Kong government.

\section{References}

Aharonian, F., Akhperjanian, A., Barrio, J., et al. 2002, A\&A, 384, L23

Akerlof, C., Balsano, R., Barthelemy, S., et al. 1999, Nature, 398, 400 Beloborodov, A. M. 2005, ApJ, 618, L13

Blumenthal, G. R., \& Gould, R. J. 1970, Rev. Mod. Phys., 42, 237

Dai, Z. G., \& Lu, T. 2002, ApJ, 580, 1013

De Jager, O. C., \& Stecker, F. W. 2002, ApJ, 566, 738

Dermer, C. D., \& Atoyan, A. 2004, A\&A, 418, L5

Fox, D. W., Price, P. A., Soderberg, A. M., et al. 2003, ApJ, 586, L5

González, M. M., Dingus, B. L., Kaneko, Y., et al. 2003, Nature, 424, 749

Granot, J., \& Guetta, D. 2003, ApJ, 598, L11

Guetta, D., \& Granot, J. 2003, ApJ, 585, 885

Panaitescu, A., \& Mészáros, P. 2000, ApJ, 544, L17

Pe'er, A., \& Waxman, E. 2004a, ApJ, 603, L1

Pe'er, A., \& Waxman, E. 2004b, ApJ, submitted [arXiv:astro-ph/0407084]

Preece, R. D., Briggs, M. S., Mallozzi, R. S., et al. 2000, ApJS, 126, 19

Kobayashi, S. 2000, ApJ, 545, 807

Kobayashi, S., \& Zhang, B. 2003, ApJ, 582, L75

Kulkarni, S. R., Frail, D. A., Sari, R., et al. 1999, ApJ, 522, L97

McMahon, E., Kumar, P., \& Panaitescu, A. 2004, MNRAS, 354, 915

Mészáros, P., \& Rees, M. J. 1993, ApJ, 418, L59

Mészáros, P., \& Rees, M. J. 1997, ApJ, 476, 232

Mészáros, P., Ramirez-Ruiz, E., Rees, M. J., \& Zhang, B. 2002, ApJ, 578, 812

Nakar, E., \& Piran, T. 2004, MNRAS, 353, 647

Nakar, E., \& Piran, T. 2005, ApJ, 619, L147

Panaitescu, A., \& Kumar, P. 2004, MNRAS, 353, 511

Rees, M. J., \& Mészáros, P. 1994, ApJ, 430, L93

Sari, R., \& Piran, T. 1995, ApJ, 455, L143

Sari, R., \& Piran, T. 1999a, ApJ, 517, L109

Sari, R., \& Piran, T. 1999b, ApJ, 520, 641

Sari, R., \& Esin, A. A. 2001, ApJ, 548, 787

Sari, R., Piran, T., \& Narayan, R. 1998, ApJ, 497, L17

Totani, T. 1998, ApJ, 502, L13

Vietri, M. 1995, ApJ, 453, 883

Wang, X. Y., Dai, Z. G., \& Lu, T. 2001a, ApJ, 546, L33

Wang, X. Y., Dai, Z. G., \& Lu, T. 2001b, ApJ, 556, 1010

Wang, X. Y., Dai, Z. G., \& Lu, T. 2003, Stellar astrophysics - A tribute to Helmut A. Abt (Kluwer Academic Publishers), ed. K. S. Cheng, K. C. Leung, \& T. P. Li [arXiv: astro-ph/0211644]

Wang, X. Y., Cheng, K. S., Dai, Z. G., \& Lu, T. 2004, ApJ, 604, 306

Waxman, E. 1995, Phys. Rev. Lett., 75, 386

Waxman, E. 1997a, ApJ, 485, L5

Waxman, E. 1997b, ApJ, 489, L33

Wei, D. M. 2003, A\&A, 402, L9

Wijers, R. A. M. J., Rees, M. J., \& Mészáros, P. 1997, MNRAS, 288, L51

Zhang, B., \& Mészáros, P. 2002, ApJ, 581, 1236

Zhang, B., Kobayashi, S., \& Mészáros, P. 2003, ApJ, 595, 950 